\title{
Perspectives on cancer and phytotherapy: an overview focusing on Polypodium leucotomos therapeutic properties
}

\begin{abstract}
Precision medicines have attempted to delivery novel individual therapy strategies for several human conditions, including cancer. Natural products from plants seem to represent an excellent resource for targeted therapies, as its phytochemicals act outbreaking multiple targets at the same time. Phytochemical and natural botanical extracts such as Polypodium leucotomos (PL), a tropical fern found in Central and South America, demonstrate a strong potential as adjuncts to the therapy of several pathologies. In the present review we aim to report the beneficial use of PL extract, including new preliminary research data from our group studies on PL and cancer disease. A systematic review of the literature was performed focusing on the main properties of PL extracts in the context of its therapeutic effects against some human conditions, demonstrated through experimental models in vitro, in vivo and in clinical trials. The phytotherapy using PL was initially introduced to the market for the treatment of inflammatory changes and other skin diseases such as vitiligo, psoriasis and skin damage caused by UV radiation. P. leucotomos contains several chemical phenolic compounds, which confers therapeutical properties such as: anti-inflammatory action, photoprotective, healing, antioxidant, immunomodulatory, photoaging prevention and antitumor activity, as lately it was described it ability to protect the cellular DNA against mutations that cause cancer. Also, PL oral supplementation acts at a molecular and cellular level to enhance endogenous antioxidant systems and inhibit generation of reactive oxygen species, thus decreasing UV-mediated oxidative DNA mutations, reducing the inflammatory responses and inhibiting extracellular matrix remodeling. The dual usage of PL, as topic cream or as a systemic protective factor, make of it a very interesting phytoterapeutical agent that would provide significant advantages such as a more uniform coverage over the body. Oral administration of PL extracts and its favorable safety profile could have significant implications in the prevention of skin, as well as others types of cancer. Altogether, the results of the studies revised clearly show the beneficial action of PL, and further exploring its role in cancer therapeutical scenario becomes very relevant for future investigations. In conclusion, the target searching of phytochemicals and phytotherapy into cancer precision medicine represents a valuable asset for new therapeutical strategies.
\end{abstract}

Volume I2 Issue I - 202 I

\author{
Pammela Araújo Lacerda, Liliane Marinho \\ Ottoni Costa, Guilherme Cuoghi Bellato, \\ Mariana Ayaka Yamashita, Lucilene Lopes- \\ Santos, Taize M Augusto, Nilva Karla \\ Cervigne
}

Faculty of Medicine of Jundiai (FMJ), São Paulo, Brazil

\begin{abstract}
Correspondence: Nilva K. Cervigne, Professor (Principal Investigator) Laboratory of Molecular Biology and Cell Culture (LBMCC), Department of Internal Medicine, Faculty of Medicine of Jundiai, Rua Francisco Telles, 250,Vila Arens II, Jundiai, São Paulo, Brazil, CEP: 13202-550, Tel +55 (II) 3395-2100 ext. 2137, Email nilva.cervigne@gmail.com
\end{abstract}

Received: January 18, 2021 | Published: January 29, 202 |

Keywords: phytotherapy, cancer, Polypodium leucotomos, therapeutical strategy

Abbreviations: PL, Polypodium leucotomos; UV, ultraviolet radiation; DNA, desoxirribonucleic acid; HPLC, high-performance liquid chromatography; CAMs, complementary and alternative medicines; MMPs, matrix metalloproteins; ECM, extracellular matrix; TIMP metallopeptidase inhibitor; TGF- $\beta$, transforming growth factor beta; ROS reactive oxygen species; MAP kinase, mitogen-activated protein kinase; NF- $\mathrm{kB}$, factor nuclear kappa B; AP-1, activator protein 1 ; CPD, cyclobutane pyrimidine dimer, GPx4, glutathione peroxidase 4; COX-2, ciclo-oxigenase-2; 8-MOP, 8-methoxypsoralen; 5-MOP, 5-methoxypsoralen; TNFs, tumor necrosis factors; iNOS, nitric oxide synthase; PMLE, polymorphic light eruption; AKs, Actinic keratosis; PDT, photodynamic therapy; OSCC, oral squamous cell carcinoma

\section{Introduction}

Cancer is a complex disease that origin of cell metabolic changes and represents a significantly public health problem all over the world. Its incidence varies in types across the occidental and oriental populations. In Brazil, the predominant type of cancer among the population is represented by non-melanoma skin cancer with $30 \%$ of the total cases of cancer with an estimated number of 5650 cases (Brazilian Institute of National Cancer, 2014).

It's important to mention that cancer cells are less specialized than normal cells and continue to divide uncontrolled. There are many biological capabilities acquired by cancer cells in human tumors (i.e. sustained proliferation, evading growth suppressors, evading apoptosis, immortality, angiogenesis induction, invasion and metastasis, genome instability and inflammation). All these characteristics are driven by each tumor microenvironment. ${ }^{1}$

Generally, options to the treatment of cancer undergoes to radiotherapy, chemotherapy, hormonal therapies (i.e. breast cancer and prostate cancer) and finally surgery. Some cases of cancers have recurrence, or either traditional treatments causes side effects. The use of non-traditional medicine (known as complementary and alternative medicines - CAMs) in association with traditional therapies has increased over the years, as routine for cancer treatment. CAMs could be described as a combination of different modalities of non-traditional medicine, with different products that are not usually considered part of a traditional treatment for cancer. According to the USA Federal Agency $^{2}$ there are several CAMs classifications. In the present review we will highlight the first category that corresponds to natural products; focusing, particularly, in the phytotherapeutic products, by which the positive effects has been currently demonstrated throughout studies involving the plant extract Polypodium leucotomos used for the treatment of many skin diseases, including skin cancer. 
It seems to us that alternative treatment, as the use of phytotherapics, could be a very potential candidate to soften side effects or be a strong candidate to stop cancer progression. For long years, men use a lot of types of complementary medicine and this use has a significantly increasing by years (use of phytotherapeutic products or nutritional supplements).

In the last five years, the practice of integrative care (or integrative oncology) has increased the survival of cancer patients rates. ${ }^{3}$ Among these practices, herbal and phytotherapy is the oldest used group of treatment (Ma et al., 2011). For years, patients make the use of plant products for self-medications with some satisfactory results. It's a common practice in Asia, Africa, Europe and America civilizations, but the risk of adverse-effects is a very relevant fact, since herbal preparations have lots of active ingredients and many of them haven't been tested with scientific rigor.

It's important to mention that between the years 1980's and 2002, the National Cancer Institute (NCI) showed that almost $70 \%$ of anticancer drugs were derived from natural products, ${ }^{4}$ but over the years was slowly overpass by the advance of synthetic pharmaceuticals in western countries. Nowadays, novels approaches to study several plants anti-tumor effects consists of examining its antioxidants compounds that have shown varying efficacy. However, phytochemical and natural botanical extracts such as Polypodium leucotomos (PL) seem to demonstrate a strong potential as adjuncts to DNA damage content protection (13). Indeed, PL extracts have various beneficial properties, including anti-inflammatory action, DNA photoprotection, immunoregulation, and anticarcinogenic potential (14-17).

In the present review we will compile a comprehensive overview of the different biological effects obtained from Polypodium leucotomos regarding its properties as a potential phytotherapeutical tool and its influence in human health overall.

\section{Method}

PubMed, EMBASE, and ScienceDirect database searches were conducted using keywords Polypodium leucotomos. Fifty-five articles in English were retrieved; 39 of 55 articles were of dermatological relevance. To focus on photoprotective properties, studies pertinent to PL use in patients with psoriasis, atopic dermatitis, vitiligo, and melasma were excluded, while articles commenting on antioxidant, immunoregulatory, anti-inflammatory, and antitumorigenic effects in the context of sunburn, photodermatoses, chronic skin damage, photoaging, and skin cancer were included $(\mathrm{n}=18$ of 55$)$. Table 1 cross-references PL photoprotective effects along with the cellular or molecular mechanisms involved and the relevant experimental studies.

Table I Summary of different studies reporting the use of Polypodium leucotomos extract

\begin{tabular}{|c|c|c|c|}
\hline Studied model & Properties of PL & PL cellular/molecular effects & References \\
\hline \multicolumn{4}{|l|}{ Clinical Trials } \\
\hline $\begin{array}{l}\text { Patients exposed to } \\
\text { sunlight treated vs. } \\
\text { non-treated with PL } \\
\text { orally }\end{array}$ & Photoprotection & $\begin{array}{l}\text { A significant reduction in positive photoprovocation and a significant } \\
\text { delay in PMLE lesions formation }\end{array}$ & Tanew et al. ${ }^{43}$ \\
\hline $\begin{array}{l}\text { Patients with high risk } \\
\text { of Malignant Melanoma } \\
\text { (MM) }\end{array}$ & $\begin{array}{l}\text { Phoprotection } \\
\text { Decrease DNA } \\
\text { mutagenesis }\end{array}$ & Increase photoprotection in patients with high risk of MM & Aguilera et al. ${ }^{32}$ \\
\hline $\begin{array}{l}\text { Bald patients } \\
\text { presenting at least two } \\
\text { Actinic Keratosis (AK) }\end{array}$ & $\begin{array}{l}\text { Photoprotecion Decrease } \\
\text { DNA mutagenesis }\end{array}$ & $\begin{array}{l}\text { Polypodium leucotomos improves Photodinamic therapy clearance and } \\
\text { decreased AKnumber and recurrence. Potential use as complementary } \\
\text { agent in the treatment of field cancerization }\end{array}$ & $\begin{array}{l}\text { Matteo Auriemma } \\
\text { et al. }{ }^{45}\end{array}$ \\
\hline $\begin{array}{l}\text { Healthy participants of } \\
\text { skin types II to III }\end{array}$ & $\begin{array}{l}\text { Anti-inflammatory } \\
\text { chemophotoprotection }\end{array}$ & $\begin{array}{l}\text { Reduction of UV-induced macrophage and neutrophil infiltration: } \\
\text { decrease in erythema in PL-treated skin; less sunburn cells, cyclobutane } \\
\text { Pyrimidine dimers, proliferating epidermal cells, and dermal mast cell } \\
\text { infiltration. }\end{array}$ & $\begin{array}{l}\text { Middelkamp-Hup } \\
\text { et al. }{ }^{15}\end{array}$ \\
\hline $\begin{array}{l}\text { Volunteers with } \\
\text { Fitzpatrick skin types } \\
\text { II to III }\end{array}$ & $\begin{array}{l}\text { Photoprotection } \\
\text { Decrease DNA } \\
\text { mutagenesis }\end{array}$ & $\begin{array}{l}\text { prevent UVA induced skin photodamage possibly by preventing UVA- } \\
\text { dependent mitochondrial DNA damage }\end{array}$ & Villa et al. 2010 \\
\hline $\begin{array}{l}\text { Healthy adult with } \\
\text { Fitzpatrick skin types } \\
\text { I to IV }\end{array}$ & Photoprotection & decreased ultraviolet-induced erythema intensity & Nestor et al. ${ }^{34}$ \\
\hline $\begin{array}{l}\text { Patients with } \\
\text { Idiopathic } \\
\text { Photodermatoses (IP) }\end{array}$ & Photoprotection & $\begin{array}{l}\text { Reduction of skin reaction and improvement of subjective symptoms, } \\
\text { leading to a significant protection of skin with IP }\end{array}$ & Caccialanza et al. ${ }^{29}$ \\
\hline $\begin{array}{l}\text { Individuals pre and } \\
\text { post exposure }\end{array}$ & Photoprotection & $\begin{array}{l}\text { Without PL, MMPI levels increased in } 7 \text { I\% of patients. With PL, MMPI } \\
\text { levels increased in } 14 \% \text { of patients. Structure of epidermis unchanged by } \\
\text { irradiation }\end{array}$ & Truchuelo et.al. ${ }^{42}$ \\
\hline \multicolumn{4}{|l|}{ In vivo } \\
\hline $\begin{array}{l}\text { Hairless albino mouse } \\
\text { model. }\end{array}$ & $\begin{array}{l}\text { Photoprotection } \\
\text { Decrease DNA } \\
\text { mutagenesis }\end{array}$ & $\begin{array}{l}\text { PL significantly reduced photoaging damage, including dermal elastosis. } \\
\text { Also showed reduction in the number of mice with skin tumors. } \\
\text { PL treatment inhibit some of the histologic damage associated with } \\
\text { photoaging of skin and appeared to contribute to a decrease in the } \\
\text { prevalence of UVB-induced skin tumors in mice. }\end{array}$ & Alcaraz et al 1999 \\
\hline
\end{tabular}


Table continued...

\begin{tabular}{|c|c|c|c|}
\hline Studied model & Properties of PL & PL cellular/molecular effects & References \\
\hline \multicolumn{4}{|l|}{ In vitro } \\
\hline $\begin{array}{l}\text { Xeroderma pigment } \\
\text { sum rodent model }\end{array}$ & $\begin{array}{l}\text { Decrease DNA } \\
\text { mutagenesis }\end{array}$ & Inhibits the UV-mediated formation of thymine dimers & Zattra et al. ${ }^{40}$ \\
\hline Albin hairless mice & $\begin{array}{l}\text { Anti-carcinogenic } \\
\text { Anti-inflammatory }\end{array}$ & $\begin{array}{l}\text { anti-tumoral properties of PL reside in its protective effect against UV } \\
\text { induced DNA damage, by Inhibiting overexpression of COX- } 2 \text { induced } \\
\text { by UV radiation }\end{array}$ & $\begin{array}{l}\text { Zattra et al. }{ }^{40} \\
\text { Rodríguez-Yanes } \\
\text { et al. }{ }^{60}\end{array}$ \\
\hline Hairless mice & $\begin{array}{l}\text { Immunoregulation } \\
\text { Antioxidant } \\
\text { photoprotection }\end{array}$ & $\begin{array}{l}\text { pretreatment with PL effectively reduced glutathione oxidation in both } \\
\text { blood and epidermis (potent systemic antioxidant effect). PL inhibited } \\
\text { UVR } \square \text { mediated Langerhans cell LC depletion. }\end{array}$ & Mulero et al. ${ }^{12}$ \\
\hline \multicolumn{4}{|l|}{ In vitro } \\
\hline Human keratinocytes & & $\begin{array}{l}\text { Pretreatment of cells with PL inhibited SSR } \square \text { mediated increase of } \\
\text { TNF } \square \alpha \text { and abrogated NO production. PL inhibited the SSR } \square \text { mediated } \\
\text { transcriptional activation of NF } \square \text { KB and API. Pretreatment with PL } \\
\text { exerted a cytoprotective effect against SSR } \square \text { induced damage, resulting in } \\
\text { increased cell survival. }\end{array}$ & Janczyk et al. ${ }^{38}$ \\
\hline $\begin{array}{l}\text { Human keratinocytes } \\
\text { and fibroblasts cells }\end{array}$ & $\begin{array}{l}\text { Cell cycle and cellular } \\
\text { integrity }\end{array}$ & Inhibition of MMP-I expression by PL in keratinocytes and fibroblasts & Philips et al. ${ }^{20}$ \\
\hline $\begin{array}{l}\text { Dermal fibroblasts and } \\
\text { Melanoma cells }\end{array}$ & $\begin{array}{l}\text { Cell cycle and cellular } \\
\text { integrity }\end{array}$ & Inhibits matrix metalloproteinases & Philips et al. ${ }^{24}$ \\
\hline $\begin{array}{l}\text { Human dermal } \\
\text { fibroblasts. }\end{array}$ & $\begin{array}{l}\text { Cell cycle and cellular } \\
\text { integrity }\end{array}$ & $\begin{array}{l}\text { PL prevents cell damage caused by VIS and IRA.Also, it reduces the } \\
\text { increase in MMP-I and cathepsin K expression VIS- and IRA-induced, } \\
\text { and curbs alterations in fibrillin I, fibrillin } 2 \text { and elastin expression. }\end{array}$ & $\begin{array}{l}\text { Zamarrón et al. } \\
2018 .\end{array}$ \\
\hline
\end{tabular}

PL, Polypodium leucotomos; PMLE, polymorphic light eruption; SSR, source of UV radiation; VIS, visible light; IRA, Infra-red radiation; DCs, dendritic cells; iNOS, inducible nitric oxide synthase; AP-I, activator protein I;TNF $\square \alpha$, tumor necrosis fator alfa; NO, nitric oxide

\section{Results}

\section{The Polypodium leucotomos}

Polypodium leucotomos (PL), scientific family name Polypodiaceae, member of the genus Polypodium (from Greek: many feet, due to the similarity of the leaf appear to have many small feet); popular synonyms are calaguala, calagualine, fern. It is a native plant to the elevated areas of Central America (700-2,500 meters), including Honduras and Guatemala. ${ }^{5}$ It also occurs in the plateaus of the Andes range in South American, where P. leucotomos species is known locally as "calaguala" and extracts of this fern are called "anapsos". It exhibits beneficial properties such anti-inflammatory, cytokine-suppressing and leukotriene-inhibiting actions.

PLaerial parts extract is rich in polyphenols with antioxidant effects. ${ }^{6}$ High-Performance Liquid Chromatography (HPLC) revealed that PL contains phenolic compounds that were separated according to their retention time. The most abundant were phenolic acids (cinannamic acid), specifically 3-methoxy-4-hydroxycinnamic acids (ferulic), 4-hydroxycinnamic acid (p-coumaric), 3,4-dihydroxycinnamic acid (caffeic), 3-methoxy-4-hydroxybenzoic acid (vanillic), 3,4-dihydroxycinnamic acid (caffeic), and 3-caffeoilquinic acid (chlorogenic). The Figure 1 represents a compilation of the phenolic compounds content of PL, according to Garcia et al. ${ }^{7}$ and Watchtel et al. ${ }^{8}$

Polypodium leucotomos extract is rich in polyphenols (including cinnamic, ferulic and chlorogenic acids), chemicals characterized by the presence of more than one phenolic group (a hydroxyl group attached to an aromatic ring), per molecule. Its antioxidant function is related to the hydroxyl group (-OH) present, attached to the aromatic ring, acting as a hydrogen atom or an electron donor, conferring the ability of this group to react with other free radicals. In addition to their antioxidant capacity, some of them exhibit chelating properties, which involves the formation of free radicals from hydrogen peroxide. The extract of this phytotherapy can be used as an oral and topical photoprotector. ${ }^{9}$

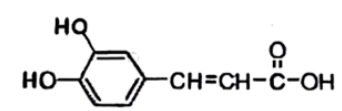

Caffeic Acid

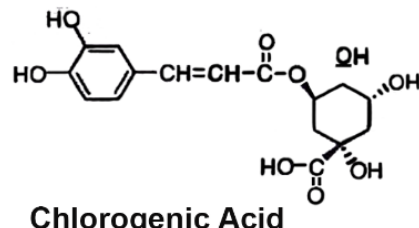

Chlorogenic Acid

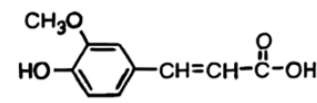

Ferulic Acid

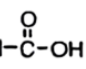

p-Coumaric Acid

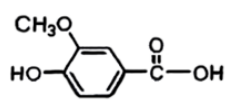

Vanillic Acid

Figure I Main compounds present in P. leucotomos. The most abundant were phenolic acids (cinannamic acid), specifically 3-methoxy-4-hydroxycinnamic acids (ferulic), 4-hydroxycinnamic acid (p-coumaric), 3,4-dihydroxycinnamic acid (caffeic), 3-methoxy-4-hydroxybenzoic acid (vanillic), 3,4-dihydroxycinnamic acid (caffeic), and 3-caffeoilquinic acid (chlorogenic). Adapted Source: L.Gombau et al., 2006.

Due to its anti-inflammatory properties, the extract of Polypodium leucotomos is used in the treatment of certain diseases involving conditions of inflammation of the skin, as in the case of vitiligo and psoriasis and in the reduction of inflammation induced by ultraviolet 
(UV) radiation due to its antioxidant activity. The compounds present in the extract act to prevent the accumulation of CPD - cyclobutane pyramidine dimers - induced by UV radiation. Studies indicate that the protection mechanism involved is related to the improvement of the DNA repair system function. ${ }^{3,10,11}$

Several hydrophilic compounds are found in the extract of Polypodium leucotomos which exhibited excellent photoprotective properties either after topically or orally administration. Rich in phenolic compounds, it also contains other acids, all of them polyphenols, with known antioxidant properties. ${ }^{6}$

The extract of Polypodium leucotomos has been in evidence in recent years in international studies. However, in Brazil, few researches in this field have been conducted.

\section{The cellular and molecular biology of Polypodium leucotomos extract}

In recent years, the interest in the properties of PL has increase, due to its antioxidant properties. ${ }^{12,13}$ PL has the ability of suppressing tumor growth and this property is related to anti-inflammatory and immunoregulatory effects. ${ }^{14} \mathrm{PL}$ also accelerates the removal of photoproducts UV-induced. ${ }^{13,15}$

Their absorption rate was studied in vitro, using Caco-2 cells in order to resemble the intestinal barrier. Their antioxidative capacity was evaluated by the luminol/ $\mathrm{H}_{2} \mathrm{O}_{2}$ assay. And their metabolism was assessed using cultured primary rat hepatocytes. The most powerful antioxidants were ferulic and caffeic acids. The PL components increased the antioxidant capacity in a concentration-dependent manner. For all tested substances, the apparent permeability was similar to human post-oral administration absorption of $70 \%-100 \%$. Coumaric, vanillic and ferulic acids were metabolized by CYP450dependent mono-oxygenases. They were partially conjugated to sulfate and glucuronic acid. ${ }^{16}$

The inflammatory effect of UVR is caused by the induction of the oxidized membrane lipids to produce arachidonic acid released that is converted by cyclooxygenase enzymes into prostaglandins. This mechanism causes the amplification of recruitment of inflammatory cells to the area. ${ }^{17}$ UVR activates AP-1 (Activator Protein-1), a transcription factor associated with cell growth, differentiation and apoptosis, which collaborate to photocarcinogenesis and the damage on ECM. AP-1 interferes with synthesis collagens and causes overexpression of matrix metalloproteinases in human skin and extracellular matrix degradation. ${ }^{18}$

Nevertheless, some authors have demonstrated beneficial actions of PL, such as the reduction of DNA damage by exposure to UVR from the sun, preventing the accumulation of pyrimidine cyclobutane dimers; ${ }^{10}$ anti-inflammatory properties, by inhibiting action of leukotrienes, and may be used in the treatment of certain diseases associated with inflammation of the skin, as in the case of vitiligo and psoriasis; ${ }^{19}$ as well as the inhibitory action of UVA-induced photoaging, protecting mitochondrial DNA against damage, and regulating the expression of MMPs (matrix metalloproteins), that are directly involved in the remodeling process of ECM, evident both in cases of photoaging and in cases of skin cancer. Also, studies have reported that cell cultures of keratinocytes and fibroblasts treated with PL showed MMP expression inhibited, while this same extract induces the production, and deposition, of new collagen fibers, especially types $\mathrm{I}$ and $\mathrm{V} .{ }^{20}$ Other studies report a significant antioxidant activity of this phytotherapic agent due to its high content of phenolic compounds, and the reduction in the erythematogenic response triggered by the process of inflammation induced by UVA radiation. ${ }^{16,21}$

The remodeling of the ECM in cancer or skin aging is caused by the reduction of ECM and/or increased expression of matrix metalloproteinases (MMPs), ${ }^{22}$ inhibition of collagen synthesis, or inhibition of TIMP. TGF- $\beta$ is a primary regulator of ECM. ${ }^{23}$ Aging skin displays lower levels of TGF- $\beta$, whereas TGF- $\beta$ levels are enhanced in cancer. UV radiation reduces the microfibrillar network in the dermis and in the epidermal-dermal layer and contributes to the appearance of aberrant elastic fibers. PL counteracts these alterations via its photoprotective, antioxidant and anti-inflammatory properties. PL exhibits a strong anti-aging effect. Additional anti-aging effects of PL include inhibition of 21 in vitro of the expression of MMP and increased expression of an endogenous metalloprotease inhibitor, TIMP. Inhibition of MMP-1 expression by PL in keratinocytes and fibroblasts has been reported. ${ }^{20}$ In fibroblasts, PL inhibited the expression of MMP-2 and simultaneously stimulated TIMPs (TIMP-1 and TIMP-2). In vitro, in melanoma cells, PL preferentially inhibited MMP-1 in an AP-1-dependent manner, which is consistent with reduced degradation of interstitial collagen, and stimulated TIMP-2, implicated in the inhibition of basement membrane remodeling. ${ }^{24}$

PL also stimulates deposition of types I and V collagen in UVirradiated fibroblasts and types I, III, and V collagen in non-irradiated fibroblasts. ${ }^{24}$ Stimulatory effect on types I and V collagen was observed in UVA- or UVB-irradiated fibroblasts, though UVB radiation decreased the level of stimulation of types I and V expression, and UVA radiation significantly counteracted the stimulation of collagen type I (COLI $\alpha 1)$ promoter activity by PL. However, UV radiation decreases the PL induction of type III collagen. These data imply that PL promotes the synthesis of types I and V collagen in UV radiation-exposed skin and the assembly of fibrillar collagens in the sun-protected skin. PL also modulates the expression of cytokines that control ECM remodeling and the biology of the cells implicated in this process. PL promotes expression of TGF- $\beta$ in non-irradiated or UV-irradiated fibroblasts but inhibits TGF- $\beta$ in melanoma cells, which may be responsible for the observed inhibition of MMP-1 expression induced by in these cells. This effect may be responsible for the observed inhibition of angiogenesis in vivo. ${ }^{24}$ In this regard, the effect of PL was largely similar to that of ascorbic acid. Stimulation of collagen expression was associated with the increase of TGF- $\beta$, whereas UVB radiation-mediated inhibition of collagen synthesis correlated with decreased expression of TGF- $\beta$. However, UV radiation did not decrease PL stimulation of TGF- $\beta$ expression, which suggests that UV and PL regulate TGF- $\beta$ expression by separate, yet related, pathways..$^{24}$ In summary, PL protects the ECM through two types of actions: one depends on its effect on ECM proteolytic enzymes and the overexpression of TIMPs, and the second one is related to the expression/assembly of structural collagens (types I, III, and V), and TGF- $\beta$ in fibroblasts. The anti-cancer effect of PL encompasses the inhibition of MMPs and stimulation of TIMPs, and decrease TGF- $\beta$ in melanoma cells.

The levels of skin collagen I, III and VII decrease in aging, which is increased by the activation of MMPs. In aging process, MMPs are upregulated but TIMP, are downregulated, with accelerated turnover ECM. ${ }^{25}$ UV-irradiation increases the expression of ROS and activation of MAP-kinase, NF- $\mathrm{kB}$ and AP-1. Both AP-1 and $\mathrm{NF}-\mathrm{\kappa B}$ are important for the balance of proliferation and apoptosis, heavily inflicted in carcinogenesis in aged human skin. In addition to aging, UV-irradiation causes mutations in p53, which can lead to skin cancers. ${ }^{25}$ In summary, PL protects against skin photoaging and 
oxidative damage due to the known molecular interaction with the intrinsic and extrinsic aging related factors.

Human skin contains many antioxidant substances and enzymes that continually assure the oxidative effect of the environment. If these substances are not present, oxidation can cause darkening, skin damage, and aging. ROS affect nuclear DNA and also produce "common" medina deletion. As is well-known, these ROS-induced DNA alterations cause the generation of $8-\mathrm{OH}-\mathrm{dG}$. This mutation accumulates during aging. ${ }^{26} \mathrm{UV}$ irradiation leads to the formation of CPD. The capacity in repairing CPD clearly decreases with age. ${ }^{27}$ This decrease in DNA damage repair capacity likely underlies the higher prevalence of skin cancer in older individuals. In addition, ROS are important factors in the process of carcinogenesis, and high levels of 8-OH-dG are found in many types of cancers. In aged skins, the alterations in the enzyme activities are not well characterized. However, disruption of Glutathione peroxidase 4 (GPx4), an enzyme implicated in antioxidative defense, displayed aging skin phenotypes, with an increase of lipid peroxidation, and with higher levels of the COX-2.

\section{Studies using Polypodium leucotomos: a new promise for human diseases treatment?}

Polypodium leucotomos has been used for the treatment of inflammatory diseases. Its effects have been beneficial in the treatment of vitiligo, since this plant has presented antioxidant, antiinflammatory and immunomodulatory properties in vitro. Gonzalez et al. ${ }^{28}$ studied twenty-one healthy volunteers (untreated or treated with oral psoralens (8-MOP or 5-MOP), who received Polypodium leucotomos extract and exposed to varying doses of natural solar radiation, with or without the ingestion of psoralens for the triggering of phototoxic reaction. Twelve patients were treated with the extract of Polypodium leucotomos, of which four received psoralens, and eight did not. The results demonstrated that the use of the extract was able to provoke a better photoprotective effect against erythema, suggesting that it could be used as a coadjuvant in the treatment of vitiligo.

Middelkamp-Hup et al. ${ }^{15}$ evaluated the acute photoprotective effect in nine participants with supplementation of PL extract $(7.5 \mathrm{mg} /$ $\mathrm{kg}$ body weight/dose). These people were exposed to artificial ultraviolet radiation before and after ingesting the PL capsules. The clinical evaluation showed a significant reduction of erythema after supplementation with the extract, showing that the oral administration of PL is interesting as an oral photoprotectant.

Caccialanza et al. ${ }^{29}$ evaluated individuals affected by the incidence of idiopathic photodermatoses, which did not respond to the usual available therapies. Twenty-six patients with polymorphous rash and two with solar urticaria participated in the study. All patients consumed $480 \mathrm{mg} /$ day of Polypodium leucotomos orally and were exposed to the sun. The skin response to sun exposure of 25 evaluable patients was compared to what had previously occurred without administration of PL. The authors observed a significant reduction in cutaneous reaction and subjective symptoms.

Solivellas \& Martin ${ }^{30}$ evaluated the infectious processes in athletes due to intensive training. The study compared athletes who took 480 $\mathrm{mg}$ of Polypodium leucotomos extract twice a day for 3 months $(\mathrm{n}=50)$ with a control group $(n=50)$, evaluating the beginning of infectious processes and recurrences during a period of 8 months (June 2010 to January 2011). The appearance of infectious processes in the group of athletes who used Polypodium leucotomos extract was lower when compared to the control group (14\% versus 56\%). Relapse in this group was observed in only one athlete (14.2\%) compared to ten athletes $(37.5 \%)$ in the control group.

Ramirez-Rosca et al. ${ }^{31}$ investigated whether daily treatment with the Polypodium leucotomos extract would reduce the use of topical corticosteroids in children and adolescents with atopic dermatitis. The study involved 105 patients receiving topical treatment corticosteroids - for eczema, aged between 2 and 17 years old. It was a six months randomized study, where they could receive either Polypodium leucotomos extract or placebo, always I addition to their standard treatment. It was observed a significant reduction in the use of oral histamine in the group that received the plant extract. Both children and preadults with the diagnosis of atopic dermatitis were favored by long-term treatment using Polypodium leucotomos extract to relieve symptoms such as inflammation and prurience.

Aguilera et al. $^{32}$ evaluated the effects of supplementation with $1080 \mathrm{mg}$ of Polypodium leucotomos in the improvement of the systemic photoprotection of 61 patients at risk of skin cancer. The results demonstrated that the administration of Polypodium leucotomos led to a significant reduction of sensitivity to the RUV in all patients.

A study carried out by Schalka et al. ${ }^{33}$ evaluated the efficacy of Polypodium leucotomos extract in erythema reduction and pigmentation after exposure to solar radiation in 20 volunteers exposed to UVB and UVA radiation by using a solar simulator. The minimal pigment dose and minimal erythematous dose were read after 2 and 24 hours of exposure, respectively. After the use of Polypodium leucotomos extract for 7, 14 and 28 days, the authors observed that the compound was effective in increasing the individual resistance to pigmentation and erythema resulting from UV radiation, and was beneficial in the treatment of pigment disorders of the skin, such as melasma.

Nestor et al. ${ }^{34}$ conducted a review study with the objective of investigating the benefits of the association of Polypodium leucotomos in the treatment for vitiligo, melasma and post-inflammatory hyperpigmentation, including a randomized placebo-controlled study. They got to the conclusion that Polypodium leucotomos, administered orally for twelve weeks, presented significant improvement of the severity of melasma in women. Other studies, combining the use of Polypodium leucotomos with psoralens and ultraviolet A and B narrow band, demonstrated promising results in the treatment of vitiligo. It was also observed that the extracts not only prevents many harmful effects associated with sunlight exposure, but also appears to provide adjuvant improvement when included in the treatment of vitiligo, melasma, and may have potential to assist in post inflammatory complexion.

Another study, also conducted by Nestor et al..$^{34}$ aimed to define the safety of this same extract and its capability to implement protection against ultraviolet radiation exposure, when administered to healthy patients, aged between 18 - 65 years, orally, twice a day, for approximately 8 weeks. Safety assessments included: physical examination, vital signs and clinical laboratory parameters such as hematology, global metabolic panel, partial thromboplastin time, and prothrombin time, which were obtained at the beginning and at the end of the study. It's evidences demonstrated there were no changes in any safety assessments. In fact, individuals from the placebo group were more likely to have more than one episode of sunburn than individuals treated with the Polypodium leucotomos extract. The authors concluded that Polypodium leucotomos extract, administrated twice a day, at a dosage of $240 \mathrm{mg}$, along sixty days, is capable of reducing the deleterious effects of UV radiation. 
Rodríguez-Yanes et al. ${ }^{35}$ reported that one dose of UV radiation leads to significant changes in the blood and skin of experimental hairless mice, but the oral administration of the Polypodium leucotomos extract reduces by $13 \%$ the number of proliferating cel; increases by $63 \%$ the number of $\mathrm{p} 53$ cells; increases plasma antioxidant capacity by $30 \%$ and strengthenes the elastic fibers structure of the dermis. In this study, the authors described that Polypodium leucotomos has several health-beneficial properties, such as antiinflammatory, brain stimulant, photoprotective, healing, antioxidant, immunomodulatory, anti-aging, as well as possible associated antitumor activity. In addition, a single molecular study of the effects of this phytotherapeutic on neoplastic cell line showed that the combination of Polypodium leucotomos with ascorbate (vitamin C) limited the growth of melanoma cells and their ability to redesign the extracellular matrix, through heightened expression of TIMP-1, the inhibitor of metalloproteinase, suggesting that ascorbate supplementation with PL may be beneficial in skin cancer therapy.

Studies in humans have shown that topical use of this extract inhibits erythema induced by exposure to solar radiation and that its use - both before and after exposure - significantly decreases the development of UVB-induced tumors in animals. Therefore, it reveals to delay lesions at the DNA level as well as at the level of immunosuppression by preventing the conversion of the urocanic acid from its trans form to cis and the elimination of the Langerhans cells. ${ }^{3,11}$

Studies have proved that oral PL has an important role in preventing erythema in UV-treated human skin, proving its capacity to restrict UV-mediated inflammation ${ }^{14,15}$ and also in psoralen plus ultraviolet A based therapy ${ }_{1}^{15}$ which is often used in inflammatory skin diseases, such as vitiligo and psoriasis. ${ }^{36,37}$ Apparently its molecular basis is due to its ability to suppress the expression of factors, such as (i) pro-inflammatory cytokines (TNF- $\alpha$ and inducible NOS (iNOS)); ${ }^{38}$ (ii) redox-sensitive transcriptional factors $\mathrm{AP}-1$ and $\mathrm{NF}-\kappa \mathrm{B} ;{ }^{14,38,39}$ and (iii) COX-2 and PGE2.40

A recent study regarding the effects of PL supplementation proved that administrated daily through oral supply, it reduces the inflammatory reaction caused by a single exposure to SSR, contributing to enhance erythematosus threshold. However, the expected effect on pigmentary reaction is yet to be validated. Whereas it's antioxidants capability should reduce the phototoxic danification and sequential death of keratinocytes from the upper epidermal layers, generating a longer holding od these cells and their skin melanosomes. ${ }^{41}$

A different study, regarding the deleterious effects of Infrared and Visible radiation (IR-VIS radiation) has been performed with Fernblock $\square$. The González group has shown its clinical efficacy when preventing the damaging effects of IR-VIS radiations. ${ }^{42}$ In vitro assays demonstrated that PL extract preserves skin cells from IR-VIS radiation as results of its antioxidant properties and its ability to promote the repair of DNA damage. In a prospective clinical trial, volunteers yield gluteal biopsy in two different moments: before and after irradiating IR-VIS (600 and $200 \mathrm{~J} / \mathrm{cm}^{2}$, respectively). A blend containing Fernblock (960mg/day) was dispensed for three weeks when, then, a new irradiation and biopsy were conducted. Histological and molecular studies showed an increase on the levels of MMP1 before and after irradiation with and without treatment. MMP1 was increased after irradiation with VIS-IR with respect to baseline in $71 \%$ of the patients, while this increase significantly dropped $(51.7 \%)$ in subjects treated with the mixture containing Fernblock. Immunohistochemistry experiments revealed that irradiation did not significantly affect the structure of the epidermis. ${ }^{42}$
Some clinical conditions emerge after cutaneous exposure to sunlight. Few examples include solar urticaria, polymorphic light eruption (PMLE), actinic prurigo and chronic actinic dermatitis. ${ }^{43,44}$ A study conducted in 2011 by Caccialanza et al. ${ }^{43}$ has addressed the PL potential to obviate the appearance of PMLE. The intake of PL extract for two weeks, promoted an important markdown in positive photoprovocation results, along with a significant setback in PMLE lesions development. Although the number of patients was small and considering that this was an open study, its results made clear that the use of PL extract in the prevention of PMLE in severely affected patients deserves further studying. Other trials, now with patients affected with PMLE, have demonstrated the valuable effects of PL. In those, patients exposed themselves to sunlight while treated with PL orally ( $480 \mathrm{mg} /$ day). The response of the skin after sunlight exposure was correlated to that occurring previously, without PL. The results exhibit a relevant and significant reduction of skin reaction and improvement of subjective symptoms. ${ }^{29,45}$

According to a study conducted by Auriemma et al. ${ }^{45} \mathrm{PL}$ extract association with photodynamic therapy (PDT) when treating patients with Actinic Keratosis (AKs) leads to an increase in clearance rate in comparison to PDT alone and it decreases recurrence rate of AKs within six months of treatment. AK is a well-known premalignant skin lesion and, although there are a number of treatments available, PDT is one of the most effectives. The main problem lies in the fact that PDT as a single therapy could promote DNA mutagenesis/ immunosuppression, which may lead to therapeutic failure and/or recurrences. Therefore the relevance of this trial must be considered very high.

A number of studies, including Middelkamp-Hup ${ }^{15}$ Nestor et al. ${ }^{34}$ and Pacifico et $a l .{ }^{46}$ have proven the beneficial effects of the association between UVB phototherapy (narrow band) and PL extract when treating Vitiligo. It is common knowledge in the dermatological field that NB-UVB induces melanocyte reservoirs to counterbalance depigmentation. Apparently, the immunomodulatory and antioxidant properties of PL extract may be what explains the better results of the association in comparison to NB-UVB therapy alone. The improvement in results with the combining therapy can reach up to $45 \%$ when PL is administrated orally after six months of treatment for Vitiligo vulgaris according to Pacifico et al. ${ }^{46}$ study. In all occasions, the positive effects seems to be more dominant in head and neck areas affected by the condition.

Still considering skin-associated disease and PL extract helpful action, another clinical study has also demonstrated the effectiveness of PL in treating melasma, a skin condition in adults arising from hypermelanosis on sun-exposed areas of the skin. Several methods of treatment are available to patients with melasma; however, developing novel targeted therapies has the potential to help the understanding of the pathogenesis, and to improve the skin condition. ${ }^{47} \mathrm{~A}$ study has shown the efficacy of PL extract for treating melasma. Female patients with melasma were randomized to be treated for 12 weeks with oral $\mathrm{PL}$ or placebo twice daily. Each patient applied sun protection factor SPF45 sunscreen daily. The group of patients treated with PL had decreased in mean Melasma area and severity index at 12 weeks of treatment with PL, whereas the placebo group did not. ${ }^{48}$

\section{Discussion}

Among the candidates for antineoplastic drugs, there are those that can be used both for the treatment and for the prevention of cancer. This class includes herbal medicines, which are medicines obtained from plants. Currently, the treatment of cancers, for the most part, is 
still considered as one of the most challenging problems in medicine, especially with regard to tumor heterogeneity, considered as one of the main villains in the resistance to anti-neoplastic treatments.

Resistance to anticancer drugs already appeared in the very early days of cancer chemotherapy more than half a century ago ${ }^{49}$ and it still hampers successful treatment of patients nowadays. ${ }^{50-54}$ Current chemotherapy protocols are based on the result of prospective, randomized, double-blind phase III studies, which results in similar clinical standard treatment guidelines. However, each tumor may behave in a different manner and the treatment success of individual patients still cannot be reliably predicted, although the statistical probability of treatment response for larger groups of patients can be estimated from the results of clinical trials. The reason is that even tumors of the same origin and histology may differ from patient to patient in their biological behavior. Even more, cells of the same tumor may be different from each there, and there is a substantial heterogeneity which greatly influences the response of tumor cell subpopulations to chemotherapy. ${ }^{55}$

The issue of drug resistance currently spread among multiple human conditions, including cancer disease, may be target by integrating the new discoveries on phytotherapy and the advance of precision medicine associated with that kind of therapy. The individualized medicine, through the use of modern technology platforms (e.g. "-omics" technologies, DNA/RNA sequencing, network pharmacology, used in studies of epigenomics, pharmacogenomics, nutrigenomics, metabolomics, etc), can be used as tool for the discovery of diverse treatment modalities such as cell disease-selective cytotoxic phytochemicals, and targeted fitochemotherapy. Also, recent developments in bioinformatics have contributed to the network pharmacology emerges as novel concept in therapy research. Phytotherapy, either by mixtures of several herbs, or isolated single compounds, exert their bioactivity by targeting multiple sites in diseased cells. A challenge for the research in the network pharmacology will certainly be to extract meaningful information from thousands of high throuput data points. Thereby, these technologies represent an integrative momentum to merge the best of two worlds: traditional medicine and clinical oncology.

Clinical experiences showed that combined therapy protocols were able to improve treatment results, but sustainable use of patients from cancer is far from reality in many cases. With multi-modal treatment options at hand, the knowledge about high probability that a specific drug would fail in a specific patient is valuable, because it allows early to switch to other more effective drugs or therapy strategies. The multi-targeted nature of drug action, resistance development, side effects on normal organs and tissues, inter-individual biological variations, as well as inter- and intra-tumoral differences have to be considered. Especially the problem of tumor heterogeneity and possibilities to tackle with genetically diverse tumor subpopulations deserve attention from our point of view. Heterogeneous tumor populations represent a main reason for the development of resistant refractory tumors. ${ }^{56-58}$

The importance of herbal medicines has become increasingly indisputable. On the other hand, in a first stage it is necessary to prove the effects of each of these plants scientifically, both in animal models and in cell culture, and finally through robust clinical trials studies. In the present review we report the beneficial usage of a powerful antioxidant, the Polypodium leucotomos extract, due to its high content of phenolic compounds. Despite topically use, this extract can also be administered orally, with proven safety. Different studies described have shown that Polypodium leucotomos extract has anti-inflammatory action and immunomodulatory activity, producing different effects on cellular immunity - both natural and specific. That seems to be due to the stimulating effect caused by Polypodium leucotomos extract in cells of the immune system ( $\mathrm{T}$ lymphocytes and natural killer cells) and its effect on the different cytokines, which gives it an immunomodulatory capacity and suggests the antiinflammatory activity, improvement of the clinical symptomatology and reducing of the relapse process in recurrent infections. ${ }^{59-61}$

As mentioned, when administered orally, P. leucotomos provides protection against the harmful effects of ultraviolet radiation, thus helping to minimize photoaging caused by sunlight, as well as the devastating effects that the same radiation can have when sensitized individuals are exposed to it. Research current going on in our laboratory sought to better determine the potential antitumor effects of PL orally administrated to mice with chemically-induced oral squamous cell carcinoma (OSCC) (data not published yet). For the first time, to this date, we demonstrate a significant difference in the number of tumors found between the PL-treated and non-treated groups, and controls. Histological examination of chemically-induced OSCC revealed a significant reduction in mild and severe dysplasia following 10 to 20 weeks of treatment with PL, suggesting that PL intaking may prevent malignant transformation of oral lesions during OSCC progression. Cell culturing and Molecular analysis revealed that several cell biological processes and genes related to tumor development and progression were modulated by PL.

In another investigation, we sought to verify the effect of the hydrophilic extract of Polypodium leucotomos in terms of photoprotection against UV-induced cell damage in human primary fibroblasts, and its antiproliferative potential in skin cancer cell lines. We found that pretreatment with PL exerted a cytoprotective effect against SSR-induced DNA damage, resulting in increased cell survival. PL efficiently prevented UV-induced morphological changes in human fibroblasts when the cells were exposed to UVA light, in a specific and dose-dependent way. Also, the photoprotection was not restricted to fibroblasts, as demonstrated by its effect on survival and proliferation of the human keratinocyte cell line HaCat (data not published yet). Additionally, we also demonstrate in these data that treating human skin cancer cell lines with PL significantly modulated the expression of SSR-mediated cell cycle genes such as p53, p21 and CDNK2A, compared with non-treated cells.

Altogether the studies reported in our revision demonstrate that PL mechanisms of action commonly include inhibition of the generation and the release of reactive oxygen species (ROS) by ultraviolet (UV) light. It also prevents UV- and ROS-induced DNA damage with inhibition of AP1 and NF- $\mathrm{kB}$ and protection of natural antioxidant enzyme systems. At the cellular level, PL decreases cellular apoptosis and necrosis mediated UV and inhibits abnormal extracellular matrix remodeling. More, PL reduces inflammation, prevents immunosuppression, activates tumor suppressor $\mathrm{p} 53$ and inhibits UVinduced cyclooxygenase-2 (COX-2) enzyme expression. In agreement with increased p53 activity, PL decreased UV radiation-induced cell proliferation. PL also prevents common deletions mitochondrial DNA damage induced by UVA, and MMP-1 expression induced Visible Light and Infrared Radiation. These cellular and molecular effects are reflected in inhibitions of carcinogenesis and photoaging.

In summary, the main goal of the present review was to expose the beneficial effects of PL with regard to the treatment of various human conditions, and particularly and more recently to highlight its potential antitumor effect, which could be quite interesting as an adjunct phytotherapeutic method associated with precision medicine 
for oncological therapies. These results serve as the scientific rationale and basis for the protection and effectiveness afforded by $P$. leucotomos for such diseases.

\section{Conclusion}

Polypodium leucotomos is a plant extract that has been considered for the treatment and prevention of several diseases, due to its potential properties just revised above. Following, this review focuses in the recent studies and clinical trials employing PL as an effective agent for the treatment and prevention of several human pathologies. Altogether the literature studies, including in vitro, in vivo and the clinical ones, as well as our original recently findings, postulate a multifactor mechanism of protection, not exclusively reliant on the antioxidant capability of PL, but strengthen the basic knowledge on the photoprotective and antiproliferative effects of this phytobotanical agent. Thus, the presented data supports PL extract as a potential new therapeutic agent for the treatment or adjuvant therapy of several human conditions, including cancer disease.

Also, integrating precision medicine into routine cancer therapy is certainly one of the predominant tasks of the next years to come. ${ }^{12}$ Particularly, Polypodium leucotomos extract has proven to be a safe and effective method, leading to significant skin protection and should be studied further, as well as its mechanisms of action and association with treatments already accepted.

This is true independent of whether synthetic drugs or phytotherapeutic approaches will be used. While there is a plethora of literature on the preclinical activity of phytochemicals and medicinal plant preparations, results from clinical trials are still relatively rare. However, there are well-done clinical trials that provide evidence that phytochemicals and plant preparations are indeed active in the clinical setting, ${ }^{53}$ specially, as an adjuvant for the current standard therapies. For the sake of future patients, health care systems in industrialized and developing countries should do any effort to improve cure rates of tumor diseases.

\section{Acknowledgments}

The authors want to dedicate this review article to all patients with cancer, specially the terminal ones, who fight every minute and try all to be one more day with their beloved ones.

Acknowledgments to the Fundação de Amparo a Pesquisa do Estado de São Paulo-FAPESP, São Paulo, Brazil (2016213477 and 2018174956), for supporting part of the research focusing on exploring Polypodium leucotomos antitumor effects, and that generated some of the original data discussed in this review.

\section{Conflicts of interest}

All the authors declare no conflict of interest.

\section{Funding}

None.

\section{References}

1. Hanahan D, Weinberg RA. Hallmarks of cancer: the next generation. Cell. 2011;144(5):646-674.

2. National Center for Complementary and Integrative Medicine (NCCIM), Complementary, Alternative, or Integrative Health: What's in a Name?, 2015 .

3. De Vries E, Arnold M, Altsitsiadis E, et al. Potential impact of interventions resulting in reduced exposure to ultraviolet (UV) radiation
(UVA and UVB) on skin cancer incidence in four European countries, 2010-2050. Br J Dermatol. 2012;167 Suppl 2:53-62.

4. Newman DJ, Cragg GM. Natural products as sources of new drugs over the last 25 years. J Nat Prod. 2007;70(3):461-477.

5. Stolze RG. Ferns and fern allies of Guatemala. Part II. Polypodiaceae, vol. 6. Field Museum of Natural History. Chicago 1981

6. Działo M, Mierziak J, Korzun U, et al. The Potential of Plant Phenolics in Prevention and Therapy of Skin Disorders. Int J Mol Sci. 2016;17(2):160.

7. García F, Pivel JP, Guerrero A, et al. Phenolic components and antioxidant activity of Fernblock, an aqueous extract of the aerial parts of the fern Polypodium leucotomos. Methods Find Exp Clin Pharmacol. 2006;28(3):157-160.

8. Wachtel-Galor S, Benzie IFF, An introduction to its history, usage, regulation, current trends, and research needs. In: Benzie IFF \& WachtelGalor S, editors. Herbal Medicine: Biomolecular and Clinical Aspects. 2nd ed. Boca Raton, FL, USA: CRC Press/Taylor \& Francis; 2011:1-10.

9. NTP (National ToxiScology Program). Report on Carcinogens, Thirteenth Edition; Department of Health and Human Services, Public Health Service: Research Triangle Park, NC, USA, 2 October 2014.

10. Nishigori C. Cellular aspects of photocarcinogenesis. Photochem Photobiol Sci. 2006;5(2):208-214.

11. Rundhaug JE, Pavone A, Kim E, et al. The effect of cyclooxygenase-2 overexpression on skin carcinogenesis is context dependent. Mol Carcinog. 2007;46(12):981-992.

12. Mulero M, Rodríguez-Yanes E, Nogues MR, et al. Polypodium leucotomos extract inhibits glutathione oxidation and prevents Langerhans cell depletion induced by UVB/UVA radiation in a hairless rat model. Exp Dermatol. 2008;17(8) 653-658.

13. Rodríguez-Yanes E, Cuevas J, González S, et al. Oral administration of Polypodium leucotomos delays skin tumor development and increases epidermal p53 expression and the anti-oxidant status of UV-irradiated hairless mice. Exp Dermatol. 2014;23(7):526-528.

14. González S, Gilaberte Y, Philips N, et al. Fernblock: A nutritional with photoprotective properties and potential preventive agent for skin photoaging and photoinduced skin cancers. Int $J$ Mol Sci. 2011;12(12):8466-8475.

15. Middelkamp-Hup MA, Pathak MA, Parrado C, et al. Oral Polypodium leucotomos extract decreases ultraviolet-induced damage of human skin. J Am Acad Dermatol. 2004;51(6):910-918.

16. Gombau L, Garcia F, Lahoz A, et al. Polypodium leucotomos extract, Antioxidant activity and disposition. Toxicol in Vitro. 2006;20(4):464471 .

17. Yaar M, Gilchrest BA. Photoageing, mechanism, prevention and therapy. Br J Dermatol. 2007;157(5):874-887.

18. Cooper SJ, Bowden GT. Ultraviolet B regulation of transcription factor families: Roles of nuclear factor-kappa B (NF-_B) and activator protein-1 (AP-1) in UVB-induced skin carcinogenesis. Curr Cancer Drug Targets. 2007;7(4):325-334.

19. Seifried HE, Anderson DE, Fisher EI, et al. A review of the interaction among dietary antioxidants and reactive oxygen species. J Nutr Biochem. 2007;18(9):567-579.

20. Philips N, Smith J, Keller T, et al. Predominant effects of Polypodium leucotomos on membrane integrity, lipid peroxidation, and expression of elastin and matrixmetalloproteinase-1 in ultraviolet radiation exposed fibroblasts, and keratinocytes. J Dermatol Sci. 2003;32(1):1-9.

21. Jansen R. Wang SQ, Burnett M, et al. Photoprotection: Part I. Photoprotection by naturally occurring, physical, and systemic agents. J Am Acad Dermatol. 2013;69(6):1-12. 
22. Gupta A, Kaur CD, Jangdey M, et al. Matrix metalloproteinase enzymes and their naturally derived inhibitors, novel targets in photocarcinoma therapy. Ageing Res Rev. 2014;13:65-74.

23. Wilkins-Port CE, Higgins PJ. Regulation of extracellular matrix remodeling following transforming growth factor-_1/epidermal growth factor-stimulated epithelial-mesenchymal transition in human premalignant keratinocytes. Cells Tissues Organs. 2007;185(1-3):116122.

24. Philips N, Conte J, Chen YJ, et al. Beneficial regulation of matrixmetalloproteinases and their inhibitors, fibrillar collagens and transforming growth factor-beta by Polypodium leucotomos, directly or in dermal fibroblasts, ultraviolet radiated fibroblasts, and melanoma cells. Arch Dermatol Res. 2009;301(7):487-495.

25. Kohl E, Steinbauer J, Landthaler M, et al. Skin ageing. J Eur Acad Dermatol. 2011;25(8):873-884.

26. Nie B, Gan W, Shi F, et al. Age-dependent accumulation of 8-oxoguanine in the DNA and RNA in various rat tissues. Oxid Med Cell Longev. $2013 ; 433-454$

27. Sauvaigo S, Caillat S, Odin F, et al. Effect of aging onDNAexcision/ synthesis repair capacities of human skin fibroblasts. $J$ Investig Dermatol. 2010;130(6):1739-1741.

28. González S, Pathak M, Cuevas J, et al. Topical or oral administration with an extract of Polypodium leucotomos prevents acute sunburn and psoralen-induced phototoxic reactions as well as depletion of Langerhans cells in human skin. Photodermatol Photoimmunol Photomed. 1997;13(1-2):50-60.

29. Caccialanza M, Percivalle S, Piccinno R, et al. Photoprotective activity of oral Polypodium Leucotomos extract in 25 patients with idiopathic photodermatoses. Photodermatol Photoimmunol Photomed. 2007;23(1):46-47.

30. Solivellas BM, Martín TC. Polypodium leucotomos Extract use to prevent and reduce the risk of infectious diseases in high performance athletes. Infect Drug Resist. 2012;5:149-153.

31. Ramírez-Bosca A, Zapater P, Betloch I, et al. Polypodium leucotomos extract in atopic dermatitis: a randomized, double-blind, placebocontrolled, multicenter trial. Actas Dermosifiliogr. 2012;103(7):599_ 607.

32. Aguilera P, Carrera C, Puig-Butille JA, et al. Benefits of oral Polypodium Leucotomos extract in MM high-risk patients. J Eur Acad Dermatol Venereol. 2013;27(9):1095-1100.

33. Schalka S, Vitale M, Agelune C, et al. The benefits of using a compound containing Polypodium leucotomos extract for reducing erythema and pigmentation resulting from ultraviolet radiation. Surg Cosmet Dermatol. 2014;6(4):344-348.

34. Nestor M, Bucay V, Callender V, et al. Polypodium leucotomos as an adjunct treatment of pigmentary disorders. J Clin Aesthet Dermatol. 2014;7(3):13-17.

35. Rodríguez-Yanes E, Juarranz A, Cuevas J, et al. Polypodium leucotomos decreases UV-induced epidermal cell proliferation and enhances p53 expression and plasma antioxidant capacity in hairless mice. Exp Dermatol. 2012;21(8):638-640.

36. Mohammad A. Vitiligo repigmentation with Anapsos (Polypodium leucotomos). Int J Dermatol. 1989;28(7):479.

37. Padilla HC, Lainez H, Pacheco JA. A new agent (hydrophilic fraction of Polypodium leucotomos) for management of psoriasis. Int J Dermatol. 1974;13(5):276-282.

38. Janczyk A, Garcia-Lopez MA, Peñas P, et al. 2007. A Polypodium leucotomos extract inhibits solar-simulated radiation-induced TNFalpha and iNOS expression, transcriptional activation and apoptosis. Exp Dermatol. 2007;16(10):823-829.
39. Bosch RP, Neena P, Jorge A, et al. Mechanisms of photoaging and cutaneous photocarcinogenesis, and photoprotective strategies with phytochemicals. Antioxidants. 2015;4(2):248-268.

40. Zattra E, Coleman C, Arad S, et al. Polypodium leucotomos extract decreases UV-induced Cox-2 expression and inflammation, enhances DNA repair, and decreases mutagenesis in hairless mice. Am J Pathol. 2009;175(5):1952-1961.

41. Calzavara-Pinton PG, Rossi MT, Zanca A, et al. Oral Polypodium leucomotos increases the anti-inflammatory and melanogenic responses of the skin to different modalities of sun exposures: A pilot study. Photodermatol Photoimmunol Photomed. 2016;32(1):22-27.

42. Truchuelo MT, Jiménez N, Mascaraque M, et al. Pilot study to assess the effects of a new oral photoprotector against infrared-visible radiations. $J$ Investig Dermatol. 2016;136:S106.

43. Tanew A, Radakovic S, González S, et al. Oral administration of a hydrophilic extract of Polypodium leucotomos for the prevention of polymorphic light eruption. J Am Acad Dermatol. 2012;66(1):58-62.

44. Caccialanza M, Recalcati S, Piccinno R, Oral Polypodium leucotomos extract photoprotective activity in 57 patients with idiopathic photodermatoses. G Ital Dermatol Venereol. 2011;146(2):85-87.

45. Auriemma M, Di Nicola M, González S, et al. Polypodium leucotomos supplementation in the treatment of scalp actinic keratosis, could it improve the efficacy of photodynamic therapy? Dermatol Surg. 2015;41(8):898-902.

46. Pacifico A, Vidoli P, Leone G, et al. Combined treatment of narrowband ultraviolet B light (NBUVB) phototherapy and oral Polypodium leucotomos extract versus NB UVB phototherapy alone in the treatment of patients with vitiligo. J Am Acad Dermatol. 2009;60(Suppl $\mathrm{S} 1): \mathrm{AB} 154$.

47. Sheth VM, Pandya AG. Melasma, a comprehensive update, part II. J Am Acad Dermatol. 2011;65(4):699-714.

48. Martin LK, Caperton C, Woolery-Lloyd H. A randomized double-blind placebo controlled study evaluating the effectiveness and tolerability of oral Polypodium leucotomos in patients with melasma. J Am Acad Dermatol. 2012;66(Supp1 S1):AB21.

49. Rhoad CP. Report on a cooperative study of nitrogen mustard (HN2) therapy of neoplastic disease. Transactions the association of American physicians. 1947;60:110-117.

50. Mellor HR, Callaghan R. Resistance to chemotherapy in cancer: a complex and integrated cellular response. Pharmacology. 2008;81(4):275-300.

51. Lin Y, Kazlova V, Ramakrishnan S, et al. Bone health nutraceuticals alter microarray mRNA gene expression: A randomized, parallel, openlabel clinical study. Phytomedicine. 2016;23(1)18-26.

52. Lippert TH, Ruoff HJ, Volm M. Could a revision of the current guidelines for cancer drug use improve the quality of cancer treatment? Ther Clin Risk Manag. 2014;10:69-72.

53. Mao JJ, Xie SX, Keefe JR, et al. Amsterdam JD. Long-term chamomile (Matricaria chamomilla L.) treatment for generalized anxiety disorder: A randomized clinical trial. Phytomedicine. 2016;23(14):1735-1742.

54. Kresty LA, Mallery SR, Stoner GD. Black raspberries in cancer clinical trials: Past, present and future. J Berry Res. 2016;6(2):251-261.

55. Weh KM, Aiyer HS, Howell AB, et al. Cranberry proanthocyanidins modulate reactive oxygen species in Barrett's and esophageal adenocarcinoma cell lines. J Berry Res. 2016;6(2):125-136.

56. Efferth T, Saeed ME, Mirghani E, et al. Integration of phytochemicals and phytotherapy into cancer precision medicine. Oncotarget. 2017;8(30):50284-50304. 
57. Cheng KC, Cahill DS, Kasai H, et al. 8-Hydroxyguanine, an abundant form of oxidative DNA damage, causes G-T and A-C substitutions. $J$ Biol Chem. 1992;267(1):166-172.

58. De la Fuente H, Tejedor R, García-López MA, et al. Polypodium leucotomos induces protection of UV-induced apoptosis in human skin cells. J Investig Dermatol. 2005;124:A121.

59. El-Haj N, Goldstein N. Sun protection in a pill, the photoprotective properties of Polypodium leucotomos extract. Int $J$ Dermatol. 2015;54(3):362-366.
60. INCA. Instituto Nacional de Câncer. Estimativa 2014: Incidência de câncer no Brasil. Rio de Janeiro: INCA, 2014. 124p.

61. Paur I, Carlsen MH, Halvorsen BL, et al. Antioxidants in herbs and spices roles in oxidative stress and redox signaling. In: Benzie IFF, Wachtel-Galor S, editors. Herbal Medicine: Biomolecular and Clinical Aspects. 2nd ed. Boca Raton, FL, USA: CRC Press/Taylor \& Francis; 2011:11-36. 